\title{
Norm Estimates for Selfadjoint Toeplitz Operators on the Fock Space
}

\section{Antonio Galbis ${ }^{1}$}

Received: 11 May 2021 / Accepted: 21 November 2021 / Published online: 16 January 2022

(c) The Author(s) 2022

\section{Abstract}

An estimate for the norm of selfadjoint Toeplitz operators with a radial, bounded and integrable symbol is obtained. This emphasizes the fact that the norm of such operator is strictly less than the supremum norm of the symbol. Consequences for time-frequency localization operators are also given.

Keywords Fock space · Toeplitz operator · Localization operator · Time-frequency

Mathematics Subject Classification 47B35 · 47G30

\section{Introduction}

The Bargmann-Fock space $\mathcal{F}^{2}(\mathbb{C})$ is the Hilbert space consisting of those analytic functions $f \in H(\mathbb{C})$ such that

$$
\|f\|_{\mathcal{F}}^{2}=\int_{\mathbb{C}}|f(z)|^{2} e^{-\pi|z|^{2}} d A(z)<+\infty
$$

where $d A(z)$ denotes the Lebesgue measure. $\mathcal{F}^{2}(\mathbb{C})$ admits a reproducing kernel $K_{w}(z)=e^{\pi \bar{w} z}$, which means that

Communicated by Vladimir Bolotnikov.

This research was partially supported by the projects MCIN

PID2020-119457GBI00/AEI/10.13039/501100011033 and GV Prometeu/2021/070.

This article is part of the topical collection "Reproducing kernel spaces and applications" edited by Daniel Alpay.

$凶$ Antonio Galbis

antonio.galbis@uv.es

1 Departament d'Anàlisi Matemàtica, Universitat de València, 46100 Burjassot, València, Spain 


$$
f(w)=\left\langle f, K_{w}\right\rangle, \quad f \in \mathcal{F}^{2}(\mathbb{C}) .
$$

The normalized monomials

$$
e_{n}(z)=\left(\frac{\pi^{n}}{n !}\right)^{\frac{1}{2}} z^{n}, \quad n \geq 0,
$$

form an orthonormal basis. For a fixed $a \in \mathbb{C}$ the translation operator

$$
W_{a}: \mathcal{F}^{2}(\mathbb{C}) \rightarrow \mathcal{F}^{2}(\mathbb{C}),\left(W_{a} f\right)(z)=f(z-a) e^{-\frac{\pi}{2}|a|^{2}+\pi z \bar{a}},
$$

is an isometry (see [14, Proposition 2.38]). We denote $d \lambda(z)=e^{-\pi|z|^{2}} d A(z)$, so $\mathcal{F}^{2}(\mathbb{C})$ is a closed subspace of $L^{2}(\mathbb{C}, d \lambda)$. The orthogonal projection

$$
P: L^{2}(\mathbb{C}, d \lambda) \rightarrow \mathcal{F}^{2}(\mathbb{C})
$$

is the integral operator

$$
(P f)(z)=\int_{\mathbb{C}} f(w) K_{w}(z) d \lambda(w)
$$

For a measurable and bounded function $F$ on $\mathbb{C}$ the Toeplitz operator with symbol $F$ is defined as

$$
T_{F}(f)(z)=P(F f)(z)=\int_{\mathbb{C}} F(w) f(w) K_{w}(z) d \lambda(w) .
$$

The systematic study of Toeplitz operators on the Fock space started in [3,4]. Since then it has been a very active research area. We refer to [14, Chapter 6], where boundedness and membership in the Schatten classes is discussed.

It is obvious that

$$
T_{F}: \mathcal{F}^{2}(\mathbb{C}) \rightarrow \mathcal{F}^{2}(\mathbb{C})
$$

is a bounded operator and

$$
\left\|T_{F}(f)\right\| \leq\|F f\|_{L^{2}(\mathbb{C}, d \lambda)} \leq\|F\|_{\infty} \cdot\|f\| .
$$

In particular, $\left\|T_{F}\right\| \leq 1$ whenever $\|F\|_{\infty} \leq 1$. If moreover $T_{F}$ is compact, which happens for instance when $F \in L^{1}(\mathbb{C})$, then $\left\|T_{F}\right\|$ is strictly less than 1 but, as far as we know, no precise estimate for the norm is known. The main result of the paper gives a bound for $\left\|T_{F}\right\|$ in the case that the symbol $F$ is radial, real-valued, and satisfies some integrability condition. For Toeplitz operators with radial symbols we refer to [11]. Besides Toeplitz operators on the Fock space we consider time-frequency localization operators with Gaussian window, also known as anti-Wick operators. They were introduced by Daubechies [7] as filters in signal analysis and can be obtained from Toeplitz operators on the Fock space after applying Bargmann transform. 


\section{Toeplitz Operators on the Fock Space}

The Toeplitz operator defined by a real valued symbol $F$ is self-adjoint. This is immediate from the identity

$$
\left\langle T_{F}(f), g\right\rangle=\int_{\mathbb{C}} F(z) f(z) \overline{g(z)} d \lambda(z)
$$

for all $f, g \in \mathcal{F}^{2}(\mathbb{C})$. In this case we have

$$
\left\|T_{F}\right\|=\sup _{\|f\|=1}\left|\left\langle T_{F}(f), f\right\rangle\right| \leq \sup _{\|f\|=1} \int_{\mathbb{C}}|F(z)| \cdot|f(z)|^{2} d \lambda(z)
$$

A symbol $F$ is said to be radial with respect to $a \in \mathbb{C}$ if $F(z)=g(|z-a|)$ for some bounded and measurable function $g$ on $[0,+\infty)$. The main result of the paper is as follows.

Theorem 1 Let $F \in L^{1}(\mathbb{C}) \cap L^{\infty}(\mathbb{C})$ be a real-valued and radial symbol with respect to $a \in \mathbb{C}$. Then

$$
\left\|T_{F}\right\| \leq\|F\|_{\infty}\left(1-\exp \left(-\frac{\|F\|_{1}}{\|F\|_{\infty}}\right)\right)
$$

An expression for the norm of Toeplitz operators with radial symbols can be found in [11] but it is unclear how the estimate provided by Theorem 1 can be obtained from it.

For the proof we will need some auxiliary results. First we observe that for $|F(z)|=$ $g(|z|)$ and $f=\sum_{n=0}^{\infty} b_{n} e_{n}$ we have, after changing to polar coordinates,

$$
\begin{aligned}
\int_{\mathbb{C}}|F(z)| \cdot|f(z)|^{2} d \lambda(z) & =\sum_{n=0}^{\infty}\left|b_{n}\right|^{2} \int_{\mathbb{C}} g(|z|)\left|e_{n}(z)\right|^{2} d \lambda(z) \\
& =\sum_{n=0}^{\infty}\left|b_{n}\right|^{2} 2 \pi \int_{0}^{\infty} g(r) \pi^{n} \frac{r^{2 n+1}}{n !} e^{-\pi r^{2}} d r \\
& =\sum_{n=0}^{\infty}\left|b_{n}\right|^{2} \int_{0}^{\infty} g\left(\sqrt{\frac{t}{\pi}}\right) \frac{t^{n}}{n !} e^{-t} d t .
\end{aligned}
$$

The $d$-dimensional Lebesgue measure of a set $\Omega \subset \mathbb{R}^{d}$ is denoted $|\Omega|$ both for $d=1$ and $d=2$.

Lemma 1 Let I $\subset[0,+\infty)$ be a measurable set with finite Lebesgue measure. Then

$$
\frac{1}{n !} \int_{I} s^{n} e^{-s} d s \leq 1-e^{-|I|}
$$


Proof (a) We first assume that $I$ is a finite union of bounded intervals. The function $h(s)=\frac{s^{n}}{n !} e^{-s}$ attains its absolute maximum at $s=n$. Then $h$ increases on $[0, n]$ and decreases on $[n,+\infty)$. We consider $a \leq n \leq b$ such that

$$
n-a=|I \cap[0, n]|, b-n=|I \cap[n,+\infty)| .
$$

Then

$$
\begin{aligned}
\frac{1}{n !} \int_{I} s^{n} e^{-s} d s & \leq \int_{a}^{b} h(s) d s=\frac{e^{-a}}{n !} \int_{0}^{b-a}(t+a)^{n} e^{-t} d t \\
& =\sum_{k=0}^{n}\left(\begin{array}{l}
n \\
k
\end{array}\right) \frac{a^{n-k}}{n !} e^{-a} \int_{0}^{|I|} t^{k} e^{-t} d t \\
& =\sum_{k=0}^{n} \frac{a^{n-k}}{(n-k) !} e^{-a} \frac{1}{k !} \int_{0}^{|I|} t^{k} e^{-t} d t \\
& \leq \sup _{0 \leq k \leq n} \frac{1}{k !} \int_{0}^{|I|} t^{k} e^{-t} d t=\int_{0}^{|I|} e^{-t} d t .
\end{aligned}
$$

For the last identity observe that

$$
\frac{1}{k !} \int_{0}^{s} t^{k} e^{-t} d t=1-e^{-s} \sum_{j=0}^{k} \frac{s^{j}}{j !}
$$

(b) For a general measurable set $I$ with finite measure the conclusion follows from part (a) and the fact that for every $\varepsilon>0$ there is a set $J$, finite union of bounded intervals, with the property that

$$
|J \backslash I|+|I \backslash J| \leq \varepsilon .
$$

Lemma 2 Let $\left(I_{k}\right)_{k=1}^{N}$ be disjoint sets with finite measure and $0 \leq \varepsilon_{k} \leq 1$ for every $1 \leq k \leq N$. Then, for every $p \in \mathbb{N}_{0}$ we have

$$
\sum_{k=1}^{N} \varepsilon_{k} \int_{I_{k}} \frac{t^{p}}{p !} e^{-t} d t \leq 1-\exp \left(-\sum_{k=1}^{N} \varepsilon_{k}\left|I_{k}\right|\right) .
$$

Proof We denote by $n$ the number of indexes $k$ such that $0<\varepsilon_{k}<1$ and we proceed by induction on $n$. For $n=0$ this is the content of Lemma 1. Let us now assume $n=1$. Let $1 \leq j \leq N$ be the coordinate with the property that $0<\varepsilon_{j}<$ and check that

$$
\psi(\varepsilon):=\sum_{k \neq j} \int_{I_{k}} \frac{t^{p}}{p !} e^{-t} d t+\varepsilon \int_{I_{j}} \frac{t^{p}}{p !} e^{-t} d t+\exp \left(-\sum_{k \neq j}\left|I_{k}\right|-\varepsilon\left|I_{j}\right|\right) \leq 1
$$


for every $0 \leq \varepsilon \leq 1$. In fact, $\psi(0) \leq 1$ and $\psi(1) \leq 1$ follow from Lemma 1 . Moreover, the critical point $\varepsilon_{0}$ of $\psi$ satisfies

$$
\int_{I_{j}} \frac{t^{p}}{p !} e^{-t} d t=\left|I_{j}\right| \exp \left(-\sum_{k \neq j}\left|I_{k}\right|-\varepsilon_{0}\left|I_{j}\right|\right)
$$

Hence

$$
\begin{aligned}
\psi\left(\varepsilon_{0}\right)= & \sum_{k \neq j} \int_{I_{k}} \frac{t^{p}}{p !} e^{-t} d t+\varepsilon_{0}\left|I_{j}\right| \exp \left(-\sum_{k \neq j}\left|I_{k}\right|-\varepsilon_{0}\left|I_{j}\right|\right) \\
& +\exp \left(-\sum_{k \neq j}\left|I_{k}\right|-\varepsilon\left|I_{j}\right|\right) \\
= & \sum_{k \neq j} \int_{I_{k}} \frac{t^{p}}{p !} e^{-t} d t+\left(1+\varepsilon_{0}\left|I_{j}\right|\right) \exp \left(-\sum_{k \neq j}\left|I_{k}\right|-\varepsilon\left|I_{j}\right|\right) .
\end{aligned}
$$

Since

$$
1+\varepsilon_{0}\left|I_{j}\right| \leq \exp \left(\varepsilon_{0}\left|I_{j}\right|\right)
$$

we conclude

$$
\psi\left(\varepsilon_{0}\right) \leq \sum_{k \neq j} \int_{I_{k}} \frac{t^{p}}{p !} e^{-t} d t+\exp \left(-\sum_{k \neq j}\left|I_{k}\right|\right) \leq 1
$$

Let us assume that the Lemma holds for $n=\ell(0 \leq \ell<N)$ and let $n=\ell+1$. We consider the function $\psi:[0,1]^{\ell+1} \rightarrow \mathbb{R}$ defined by

$$
\psi(\boldsymbol{\varepsilon}):=\sum_{k=1}^{\ell+1} \varepsilon_{k} \int_{I_{k}} \frac{t^{p}}{p !} e^{-t} d t+\sum_{j} \int_{J_{j}} \frac{t^{p}}{p !} e^{-t} d t+\exp \left(-\sum_{k} \varepsilon_{k}\left|I_{k}\right|-\sum_{j}\left|J_{j}\right|\right)
$$

for $\boldsymbol{\varepsilon}=\left(\varepsilon_{1}, \ldots, \varepsilon_{\ell+1}\right)$. The induction hypothesis means that $\psi(\boldsymbol{\varepsilon}) \leq 1$ whenever $\boldsymbol{\varepsilon}$ is in the boundary of $[0,1]^{\ell+1}$. The lemma is proved after checking that $\psi\left(\varepsilon_{0}\right) \leq 1$, where $\boldsymbol{\varepsilon}_{0}$ is a critical point of $\psi$. Proceeding as before,

$$
\begin{aligned}
\psi\left(\boldsymbol{\varepsilon}_{0}\right) & =\left(\sum_{k=1}^{\ell+1} \varepsilon_{k}\left|I_{k}\right|+1\right) e^{-\sum_{k} \varepsilon_{k}\left|I_{k}\right|} e^{-\sum_{j}\left|J_{j}\right|}+\sum_{j} \int_{J_{j}} \frac{t^{p}}{p !} e^{-t} d t \\
& \leq \exp \left(-\sum_{j}\left|J_{j}\right|\right)+\sum_{j} \int_{J_{j}} \frac{t^{p}}{p !} e^{-t} d t \leq 1 .
\end{aligned}
$$


Proof of Theorem 1 We first assume $a=0$, that is, $F$ is radial. After replacing $F$ by $G=\frac{F}{\|F\|_{\infty}}$ if necessary we can assume that $\|F\|_{\infty}=1$. Since $F$ is radial we have $F(z)=g(|z|)$. We aim to prove that

$$
\int_{\mathbb{C}}|g(|z|)| \cdot|f(z)|^{2} e^{-\pi|z|^{2}} d A(z) \leq 1-\exp \left(-2 \pi \int_{0}^{\infty} r|g(r)| d r\right)
$$

for every entire function $f(z)=\sum_{p=0}^{\infty} b_{p} e_{p}$ such that $\sum_{p=0}^{\infty}\left|b_{p}\right|^{2}=1$. We have

$$
\int_{\mathbb{C}}|g(|z|)| \cdot|F(z)|^{2} e^{-\pi|z|^{2}} d A(z)=\sum_{p=0}^{\infty}\left|b_{p}\right|^{2} \int_{0}^{\infty}\left|g\left(\sqrt{\frac{t}{\pi}}\right)\right| \cdot \frac{t^{p}}{p !} e^{-t} d t .
$$

Let us first assume

$$
g=\sum_{k=1}^{N} \varepsilon_{k} \chi_{I_{k}},\left|\varepsilon_{k}\right| \leq 1
$$

where $\left(I_{k}\right)_{k=1}^{N}$ are disjoint intervals. Then, Lemma 1 gives

$$
\begin{aligned}
\sum_{p=0}^{\infty}\left|b_{p}\right|^{2} \int_{0}^{\infty}\left|g\left(\sqrt{\frac{t}{\pi}}\right)\right| \cdot \frac{t^{p}}{p !} e^{-t} d t & \leq 1-\exp \left(-\sum_{k=1}^{N}\left|\varepsilon_{k}\right|\left|J_{k}\right|\right) \\
& =1-\exp \left(-2 \pi \int_{0}^{\infty} r|g(r)| d r\right) \\
& =1-\exp \left(-\|F\|_{1}\right) .
\end{aligned}
$$

We used $J_{k}=\left\{t: \sqrt{\frac{t}{\pi}} \in I_{k}\right\}$ and $\left|J_{k}\right|=2 \pi \int_{I_{k}} r d r$. Theorem 1 is proved for $g$ as in (1). Let us now assume that $\|g\|_{\infty} \leq 1$ and $g \in L^{1}\left(\mathbb{R}^{+}, r d r\right) \cap L^{\infty}\left(\mathbb{R}^{+}\right)$. Then there is a sequence $\left(g_{n}\right)_{n}$ of step functions as in (1) such that

$$
\lim _{n \rightarrow \infty} \int_{0}^{\infty}\left|g_{n}(r)-g(r)\right| r d r=0
$$

We put $F_{n}(z):=g_{n}(|z|)$. According to [12, Theorem 3.5] there is a constant $K>0$ such that

$$
\left\|T_{G}\right\| \leq K \sup _{z \in \mathbb{C}} \int_{D(z, 1)}|G| d A
$$

for every bounded symbol $G$, which implies 


$$
\lim _{n \rightarrow \infty}\left\|T_{F}-T_{F_{n}}\right\| \leq K \lim _{n \rightarrow \infty}\left\|F_{n}-F\right\|_{1}=0
$$

We finally conclude

$$
\left\|T_{F}\right\| \leq 1-\exp \left(-\|F\|_{1}\right)
$$

In the case $a \neq 0$, the identity

$$
\int_{\mathbb{C}} g(|z-a|)|f(z)|^{2} d \lambda(z)=\int_{\mathbb{C}} g(|u|)\left|\left(W_{-a} f\right)(u)\right|^{2} d \lambda(u)
$$

and the fact that $W_{-a}$ is an isometry gives the conclusion. We can also argue from the fact that $W_{-a} \circ T_{F}=T_{G} \circ W_{-a}$, where $G(z)=g(|z|)$.

In particular, if $\Omega \subset \mathbb{C}$ presents radial symmetry with respect to some point then

$$
\int_{\Omega}|f(z)|^{2} d \lambda(z) \leq\left(1-e^{-|\Omega|}\right) \cdot \int_{\mathbb{C}}|f(z)|^{2} d \lambda(z)
$$

for every $f \in \mathcal{F}^{2}(\mathbb{C})$.

The question arises whether inequality (3) holds for every subset $\Omega$. This is related to a conjecture by Abreu and Speckbacher in [1] (see the next section). We do not have an answer to this question except for monomials or its translates.

Example 1 Let $k_{w}=e^{-\frac{\pi}{2}|w|^{2}} K_{w}$ be the normalized reproducing kernel of $\mathcal{F}^{2}(\mathbb{C})$. Then, for every set $\Omega \subset \mathbb{C}$ with finite measure we have

$$
\int_{\Omega}\left|k_{w}(z)\right|^{2} d \lambda(z) \leq 1-e^{-|\Omega|}
$$

Proof In fact, $k_{w}=W_{w}\left(e_{0}\right)$. Hence

$$
\int_{\Omega}\left|k_{w}(z)\right|^{2} d \lambda(z)=\int_{\Omega-w} d \lambda(z)
$$

and the conclusion follows from the fact that the last integral attains its maximum when $\Omega$ is a disc centered at $w$ (see the comment after [1, Conjecture 1]).

It is easy to check that when $\Omega$ is a disc centered at point $\omega$ the inequality in Example 1 is an identity.

Proposition 1 Let $\Omega \subset \mathbb{R}^{2}$ be a set with finite measure. Then, for every $n \in \mathbb{N}$ and $a \in \mathbb{C}$, 


$$
\int_{\Omega}\left|W_{a}\left(e_{n}\right)(z)\right|^{2} d \lambda(z) \leq 1-e^{-|\Omega|}
$$

\section{Proof Since}

$$
\int_{\Omega}\left|W_{a}\left(e_{n}\right)(z)\right|^{2} d \lambda(z)=\int_{\Omega-a}\left|e_{n}(z)\right|^{2} d \lambda(z)
$$

we can assume that $a=0$. For every $\theta \in[0,2 \pi]$ we denote

$$
\Omega_{\theta}=\left\{r \geq 0: r e^{i \theta} \in \Omega\right\}
$$

Then

$$
\begin{aligned}
\int_{\Omega}\left|e_{n}(z)\right|^{2} d \lambda(z) & =\frac{\pi^{n}}{n !} \int_{\Omega}\left|z^{n}\right|^{2} e^{-\pi|z|^{2}} d A(z) \\
& =\frac{\pi^{n}}{n !} \int_{0}^{2 \pi}\left(\int_{\Omega_{\theta}} r^{2 n} e^{-\pi r^{2}} 2 \pi r d r\right) \frac{d \theta}{2 \pi} \\
& =\int_{0}^{2 \pi}\left(\int_{I_{\theta}} \frac{t^{n}}{n !} e^{-t} d t\right) \frac{d \theta}{2 \pi}
\end{aligned}
$$

where

$$
I_{\theta}=\left\{t=\pi r^{2}: r \in \Omega_{\theta}\right\} .
$$

Since $|\Omega|<\infty$ then a.e. $\theta \in[0,2 \pi]$ we have

$$
\left|I_{\theta}\right|=2 \pi \int_{\Omega_{\theta}} r d r<+\infty
$$

Moreover, by Lemma 1,

$$
\int_{0}^{2 \pi}\left(\int_{I_{\theta}} \frac{t^{n}}{n !} e^{-t} d t\right) \frac{d \theta}{2 \pi} \leq \int_{0}^{2 \pi}\left(1-e^{-\left|I_{\theta}\right|}\right) \frac{d \theta}{2 \pi} .
$$

Finally we consider the convex function $f(t)=e^{-t}-1$ and the probability measure $\frac{d \theta}{2 \pi}$ and put $h(\theta)=\left|I_{\theta}\right|$. Jensen's inequality gives

$$
f\left(\int_{0}^{2 \pi} h(\theta) \frac{d \theta}{2 \pi}\right) \leq \int_{0}^{2 \pi} f(h(\theta)) \frac{d \theta}{2 \pi},
$$

which means 


$$
\begin{aligned}
\int_{0}^{2 \pi}\left(1-e^{-\left|I_{\theta}\right|}\right) \frac{d \theta}{2 \pi} & \leq 1-\exp \left(-\int_{0}^{2 \pi}\left|I_{\theta}\right| \frac{d \theta}{2 \pi}\right) \\
& =1-\exp \left(-\int_{0}^{2 \pi}\left(\int_{\Omega_{\theta}} r d r\right) d \theta\right) \\
& =1-e^{-|\Omega|}
\end{aligned}
$$

We finish the section with some examples of sets $\Omega$ with infinite Lebesgue measure for which the Toeplitz operator with symbol $F=\chi_{\Omega}$ has norm as small as we want.

Proposition 2 For every $\varepsilon>0$ there exists $\Omega$ with infinite Lebesgue measure such that

$$
\int_{\Omega}|f(z)|^{2} d \lambda(z) \leq \varepsilon \int_{\mathbb{C}}|f(z)|^{2} d \lambda(z)
$$

for every $f \in \mathcal{F}^{2}$.

Proof Let $K>0$ as in (2) and let $\left(\Omega_{n}\right)_{n}$ be a sequence of bounded sets with Lebesgue measure $\left|\Omega_{n}\right|=\frac{\varepsilon}{K}$ and such that $\operatorname{dist}\left(\Omega_{n}, \Omega_{m}\right)>2$ whenever $n \neq m$, and take $\Omega=$ $\cup_{n \in \mathbb{N}} \Omega_{n}$. Since each disc $D(z, 1)$ meets at most one set $\Omega_{n}$ we have $|\Omega \cap D(z, 1)| \leq \frac{\varepsilon}{K}$. The estimate (2) turns out $\left\|T_{\chi \Omega}\right\| \leq \varepsilon$, which gives the conclusion.

\section{Time-Frequency Localization Operators}

For $F \in L^{1}(\mathbb{C})$ we denote by $H_{F}: L^{2}(\mathbb{R}) \rightarrow L^{2}(\mathbb{R})$ the localization operator

$$
H_{F} f=\int_{\mathbb{C}} F(z)\left\langle f, \pi(z) h_{0}\right\rangle \pi(z) h_{0} d A(z) .
$$

Here $h_{0}(t)=2^{1 / 4} e^{-\pi t^{2}}$ is the Gaussian and $\pi(z)$ is the time-frequency shift, defined for $z=x+i \omega$ as

$$
(\pi(z) f)(t)=e^{2 \pi i \omega t} f(t-x), f \in L^{2}(\mathbb{R}) .
$$

In case $F$ is the characteristic function of a set $\Omega$ we write $H_{\Omega}$ instead of $H_{\chi_{\Omega}}$. We refer to [5] or [6, Chapter 4] for general facts concerning localization operators.

For $f, g \in L^{2}(\mathbb{R})$, the expression

$$
\left(V_{g} f\right)(z):=\langle f, \pi(z) g\rangle
$$

is the short time Fourier transform of $f$ with window $g$, known as Gabor transform in the case where the window $g=h_{0}$ is the Gaussian.

If $F$ is real-valued then $H_{F}$ is a selfadjoint operator on $L^{2}(\mathbb{R})$, hence

$$
\left\|H_{F}\right\|=\sup _{\|f\|_{2}=1}\left|\left\langle H_{F} f, f\right\rangle\right| \leq \sup _{\|f\|_{2}=1} \int_{\mathbb{C}}|F(z)| \cdot\left|\left(V_{h_{0}} f\right)(z)\right|^{2} d A(z) .
$$


There is a connection between localization operators and Toeplitz operators on the Fock space via the Bargmann transform.

The Bargmann transform is the surjective and unitary operator

$$
\mathcal{B}: L^{2}(\mathbb{R}) \rightarrow \mathcal{F}^{2}(\mathbb{C})
$$

defined as

$$
(\mathcal{B} f)(z)=2^{1 / 4} \int_{\mathbb{R}} f(t) e^{2 \pi t z-\pi t^{2}-\frac{\pi}{2} z^{2}} d t
$$

It was introduced in [2] and has the important property that the Hermite functions are mapped into normalized analytic monomials. More precisely, $\mathcal{B}\left(h_{n}\right)=e_{n}$, where $h_{n}$ is defined via the so called Rodrigues formula as

$$
h_{n}(t)=\frac{2^{1 / 4}}{\sqrt{n !}}\left(\frac{-1}{2 \sqrt{\pi}}\right)^{n} e^{\pi t^{2}} \frac{d^{n}}{d t^{n}}\left(e^{-2 \pi t^{2}}\right), \quad n \geq 0 .
$$

Then $\left(h_{n}\right)_{n \geq 0}$ forms an orthonormal basis for $L^{2}(\mathbb{R})$. The Gabor transform of Hermite functions is well-known (see for instance [9, Chapter 1.9]). In fact, for $z=x+i \xi$,

$$
\left\langle h_{n}, \pi(z) h_{0}\right\rangle=e^{-i \pi x \xi-\frac{\pi}{2}|z|^{2}} \sqrt{\frac{\pi^{n}}{n !}} \bar{z}^{n} .
$$

Since for $z=x+i \xi$ we have $([10,3.4 .1])$

$$
\left(V_{h_{0}} f\right)(x,-\xi)=e^{i \pi x \xi} \cdot(\mathcal{B} f)(z) \cdot e^{-\frac{\pi|z|^{2}}{2}}
$$

then, for every $f \in L^{2}(\mathbb{R})$ and $F \in L^{1}(\mathbb{C}) \cap L^{\infty}(\mathbb{C})$ we obtain

$$
\int_{\mathbb{C}}|F(z)| \cdot\left|\left(V_{h_{0}} f\right)(z)\right|^{2} d A(z)=\int_{\mathbb{C}}|F(z)| \cdot|(B f)(z)|^{2} d \lambda(z) .
$$

Consequently, all the estimates in the previous section can be translated into estimates concerning localization operators.

Abreu, Speckbacher conjecture in [1] that, among all the sets with a given measure, $\left\|H_{\Omega}\right\|$ attains its maximum when $\Omega$ is a disc, up to perturbations of Lebesgue measure zero. This turns out to be equivalent to the validity of inequality (3) for every function in the Fock space or, equivalently, to the fact that

$$
\|f\|_{2}^{2} \leq e^{|\Omega|} \int_{\mathbb{C} \backslash \Omega}\left|\left(V_{h_{0}} f\right)(z)\right|^{2} d A(z) \forall f \in L^{2}(\mathbb{R}) .
$$


In this regard it is worth noting that Nazarov [13] proved the existence of two absolute constants $A, B$ such that

$$
\|f\|_{2}^{2} \leq A e^{B \cdot|S| \cdot|\Sigma|}\left(\int_{\mathbb{R} \backslash S}|f|^{2}+\int_{\mathbb{R} \backslash \Sigma} \mid \widehat{f}^{2}\right)
$$

for every $f \in L^{2}(\mathbb{R})$ and for any pair $(S, \Sigma)$ of sets with finite measure. Also, it follows from [8, Theorem 4.1] that for every set $\Omega \subset \mathbb{R}^{2}$ thin at infinity and for every $g \in L^{2}(\mathbb{R})$ there exist a constant $C>0$ such that

$$
\|f\|_{2}^{2} \leq C \int_{\mathbb{C} \backslash \Omega}\left|\left(V_{g} f\right)(z)\right|^{2} d A(z) \forall f \in L^{2}(\mathbb{R}) .
$$

From Theorem 1 and Proposition 1 we get the following.

Corollary 1 Let $F \in L^{1}(\mathbb{C}) \cap L^{\infty}(\mathbb{C})$ be a real-valued and radial symbol with respect to $a \in \mathbb{C}$. Then

$$
\left\|H_{F}\right\| \leq\|F\|_{\infty}\left(1-\exp \left(-\frac{\|F\|_{1}}{\|F\|_{\infty}}\right)\right)
$$

Corollary 2 Let $\Omega \subset \mathbb{R}^{2}$ be a set with finite measure. Then, for every $n \in \mathbb{N}$,

$$
\left|\left\langle H_{\Omega} h_{n}, h_{n}\right\rangle\right| \leq 1-e^{-|\Omega|} .
$$

We fix a non-zero window $g \in L^{2}(\mathbb{R})$. The modulation space $M^{1}(\mathbb{R})$, also known as Feichtinger algebra, is the set of tempered distributions $f \in \mathcal{S}^{\prime}(\mathbb{R})$ such that

$$
\|f\|_{M^{1}}:=\int_{\mathbb{C}}|\langle f, \pi(z) g\rangle| d A(z)<+\infty .
$$

The use of different windows $g$ in the definition of $M^{1}(\mathbb{R})$ yields the same spaces with equivalent norms. It is well known that $M^{1}(\mathbb{R})$ is continuously included in $L^{2}(\mathbb{R})$ and

$$
\|f\|_{2}=\left\|V_{g} f\right\|_{2} \leq\left\|V_{g} f\right\|_{1}
$$

whenever $f \in M^{1}(\mathbb{R})$ and $\|g\|_{2}=1$. See for instance [10, 3.2.1] for the first identity. Proposition 3 Let $\Omega \subset \mathbb{R}^{2}$ be a set with finite measure. Then, for every $f \in M^{1}(\mathbb{R})$ and $n \in \mathbb{N}_{0}$ we have

$$
\int_{\Omega}\left|\left(V_{h_{0}} f\right)(z)\right|^{2} d A(z) \leq\left\|V_{h_{n}} f\right\|_{1}^{2} \cdot\left(1-e^{-|\Omega|}\right) .
$$

Proof It suffices to prove the proposition under the additional assumption that $\left\|V_{h_{n}} f\right\|_{1}=1$. Fixed $n \in \mathbb{N}_{0}$ we consider the set

$$
B:=\left\{\pi(z) h_{n}: z \in \mathbb{C}\right\} \subset L^{2}(\mathbb{R}) .
$$


Then

$$
B^{\circ}:=\left\{g \in L^{2}(\mathbb{R}):\left|\left\langle g, \pi(z) h_{n}\right\rangle\right| \leq 1\right\}=\left\{g \in L^{2}(\mathbb{R}):\left\|V_{h_{n}} g\right\|_{\infty} \leq 1\right\} .
$$

We have

$$
|\langle f, g\rangle|=\left|\left\langle V_{h_{n}} f, V_{h_{n}} g\right\rangle\right| \leq\left\|V_{h_{n}} f\right\|_{1} \cdot\left\|V_{h_{n}} g\right\|_{\infty} \leq 1
$$

for every $g \in B^{\circ}$, which means that $f \in B^{\circ \circ}$. According to the bipolar theorem,

$$
f=L^{2}-\lim _{k \rightarrow \infty} f_{k}
$$

where each $f_{k}$ is in the absolutely convex hull of $B$. For each $k \in \mathbb{N}$ we can find scalars $\left(\alpha_{j}\right)_{j=1}^{N}$ and points $\left(z_{j}\right)_{j=1}^{N}$ such that $f_{k}=\sum_{j=1}^{N} \alpha_{j} \pi\left(z_{j}\right) h_{n}$ and $\sum_{j=1}^{N}\left|\alpha_{j}\right| \leq 1$. Then

$$
\begin{aligned}
\left(\int_{\Omega}\left|\left(V_{h_{0}} f_{k}\right)(z)\right|^{2} d A(z)\right)^{\frac{1}{2}} & =\left(\int_{\Omega}\left|\left\langle f_{k}, \pi(z) \varphi\right\rangle\right|^{2} d A(z)\right)^{\frac{1}{2}} \\
& \leq \sum_{j=1}^{N}\left|\alpha_{j}\right|\left(\int_{\Omega}\left|\left\langle\pi\left(z_{j}\right) h_{n}, \pi(z) \varphi\right\rangle\right|^{2} d A(z)\right)^{\frac{1}{2}} \\
& =\sum_{j=1}^{N}\left|\alpha_{j}\right|\left(\int_{\Omega}\left|\left\langle h_{n}, \pi\left(z-z_{j}\right) \varphi\right\rangle\right|^{2} d A(z)\right)^{\frac{1}{2}} \\
& =\sum_{j=1}^{N}\left|\alpha_{j}\right|\left(\int_{\Omega-z_{j}}\left|\left\langle h_{n}, \pi(z) \varphi\right\rangle\right|^{2} d A(z)\right)^{\frac{1}{2}} \\
& =\sum_{j=1}^{N}\left|\alpha_{j}\right|\left|\left\langle H_{\Omega-z_{j}} h_{n}, h_{n}\right\rangle\right|^{\frac{1}{2}} \leq\left(1-e^{-|\Omega|}\right)^{\frac{1}{2}} .
\end{aligned}
$$

Finally,

$$
\int_{\Omega}\left|\left(V_{h_{0}} f\right)(z)\right|^{2} d A(z)=\lim _{k \rightarrow \infty} \int_{\Omega}\left|\left(V_{h_{0}} f_{k}\right)(z)\right|^{2} d A(z) \leq 1-e^{-|\Omega|} .
$$

The next result is a direct consequence of Proposition 2.

Corollary 3 For every $\varepsilon>0$ there exists $\Omega$ with infinite Lebesgue measure such that

$$
\left\|H_{\Omega}\right\| \leq \varepsilon .
$$


Funding Open Access funding provided thanks to the CRUE-CSIC agreement with Springer Nature.

Data Availability Data sharing not applicable to this article as no datasets were generated or analysed during the current study.

\section{Declarations}

Conflict of interest The authors declare that they have no conflict of interest.

Open Access This article is licensed under a Creative Commons Attribution 4.0 International License, which permits use, sharing, adaptation, distribution and reproduction in any medium or format, as long as you give appropriate credit to the original author(s) and the source, provide a link to the Creative Commons licence, and indicate if changes were made. The images or other third party material in this article are included in the article's Creative Commons licence, unless indicated otherwise in a credit line to the material. If material is not included in the article's Creative Commons licence and your intended use is not permitted by statutory regulation or exceeds the permitted use, you will need to obtain permission directly from the copyright holder. To view a copy of this licence, visit http://creativecommons.org/licenses/by/4.0/.

\section{References}

1. Abreu, L.D., Speckbacher, M.: Donoho-Logan large sieve principles for modulation and polyanalytic Fock spaces. Bull. Sci. Math. 171, 103032 (2021)

2. Bargmann, V.: On a Hilbert space of analytic functions and an associated integral transform. Comm. Pure Appl. Math. 14, 187-214 (1961)

3. Berger, C.A., Coburn, L.A.: Toeplitz operators and quantum mechanics. J. Funct. Anal. 68, 273-299 (1986)

4. Berger, C.A., Coburn, L.A.: Toeplitz operators on the Segal-Bargmann space. Trans. Am. Math. Soc. 301, 813-829 (1987)

5. Cordero, E., Gröchenig, K.: Time-frequency analysis of localization operators. J. Funct. Anal. 205, 107-131 (2003)

6. Cordero, E., Rodino, L.: Time-Frequency Analysis of Operators. De Gruyter Studies in Mathematics, p 75 (2020)

7. Daubechies, I.: Time-frequency localization operators: a geometric phase space approach. IEEE Trans. Inform. Theory 34(4), 605-612 (1988)

8. Fernández, C., Galbis, A.: Annihilating sets for the short time Fourier transform. Adv. Math. 224, 1904-1926 (2010)

9. Folland, G.B.: Harmonic Analysis in Phase Space, vol. 122. Princeton University Press, Princeton, N.J. (1989)

10. Gröchenig, K.: Foundations of Time-Frequency Analysis. Birkhäuser, New York (2001)

11. Grudsky, S.M., Vasilevski, N.L.: Toeplitz operators on the Fock space: radial component effects. Integral Equ. Oper. Theory 44, 10-37 (2002)

12. Hu, Z.J., Lv, X.F.: Toeplitz operators on fock spaces $F^{p}(\varphi)$. Integr. Equ. Oper. Theory 80, 33-59 (2014)

13. Nazarov, F.L.: Local estimates for exponential polynomials and their applications to inequalities of the uncertainty principle type. (Russian) Algebra i Analiz 5: 3-66; translation in St. Petersburg Math. J. 5(1994), 663-717 (1993)

14. Zhu, K.: Analysis on Fock spaces. Graduate Texts in Mathematics, vol. 263. p. x+344, (2012). Springer, New York

Publisher's Note Springer Nature remains neutral with regard to jurisdictional claims in published maps and institutional affiliations. 